\title{
Plasma lipidomics are associated with adiposity in overweight or obese but otherwise healthy non-diabetic adults
}

Aya Mousa ${ }^{1}$, Negar Naderpoor $^{1,2}$, Estifanos Baye ${ }^{1}$, Josphin Johnson ${ }^{1}$, Natalie Mellett ${ }^{3}$, Peter Meikle ${ }^{3}$, Research and Implementation Barbora de Courten ${ }^{1,2}$

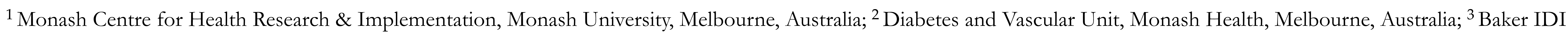
Heart and Diabetes Institute, Melbourne, Australia. Correspondence: barbora.decourten@monash.edu

\section{Background}

- Adiposity is a major risk factor for dyslipidaemia and chronic diseases including type 2 diabetes and cardiovascular disease.

- Novel lipidomics methods are providing new insights into the role of lipid metabolism in chronic diseases.

- Limited studies have examined the relationship between adiposity and the plasma lipidome in humans.

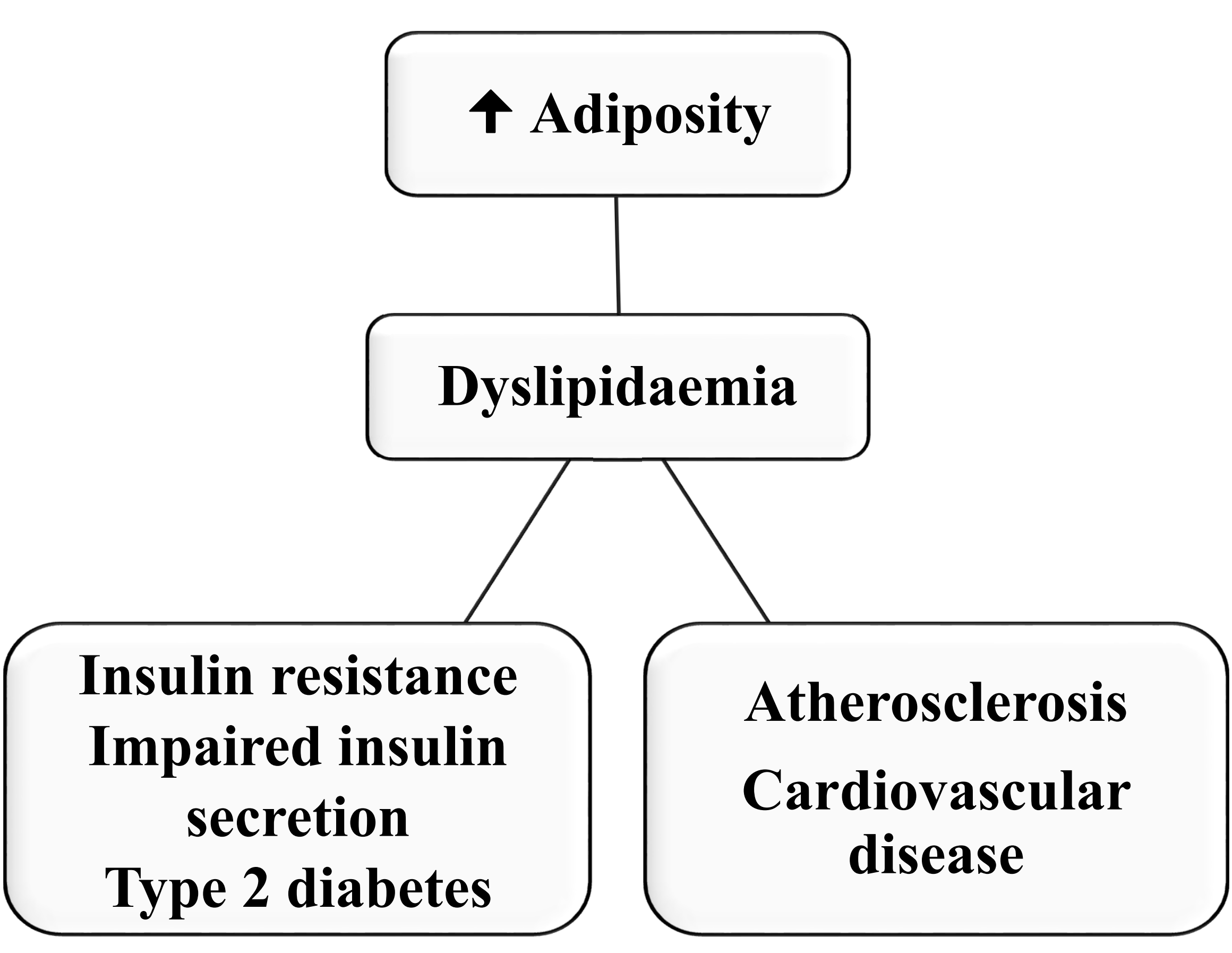

\section{Hypothesis}

We hypothesized that increased total and central adiposity will be associated with dysregulation of certain lipid species or classes.

\section{$\underline{\operatorname{Aim}}$}

To examine whether adiposity measured by body mass index (BMI), waist circumference (WC), waist-tohip ratio (WHR), or total percent body fat is associated with lipid species or classes in overweight or obese non-diabetic adults.

\section{Study population}

- 65 participants (41M / 24F):

- healthy, non-diabetic adults (WHO guidelines)

- age between 18 and 60 yrs

- overweight or obese $(\mathrm{BMI} \geq$ $25 \mathrm{~kg} / \mathrm{m}^{2}$ )

- non-smokers and not taking medications or using illicit drugs

- no chronic diseases or clinical or laboratory signs of acute or chronic inflammation

\section{Methods}

- Anthropometry: BMI, WC, WHR, and total percent body fat by dual X-ray absorptiometry (DEXA).

- Lipidomics analysis: Liquid chromatography electrospray ionization tandem mass spectrometry was used, which identified 459 lipid species across 26 lipid classes from the sphingolipid, glycerolipid, phospholipid, plasmalogen, and sterol lipid groups.

- Statistical analyses were performed on Matlab software. All analyses were adjusted for multiple testing using Benjamini Hochberg (BH) correction.

\section{$\underline{\text { Results }}$}

Table 1. Sample characteristics

\begin{tabular}{lc}
\hline & Mean \pm SD or $\mathbf{n}(\%)$ \\
\hline Male/ Female, $n$ & $41 / 24$ \\
\hline Age (years) & $31.3 \pm 8.5$ \\
BMI $\left(\mathrm{kg} / \mathrm{m}^{2}\right)$ & $31.5 \pm 5.3$ \\
WHR & $1.1 \pm 1.2$ \\
Body fat $(\%)$ & $40.1 \pm 8.7$ \\
\hline
\end{tabular}

- On univariable analyses, total percent body fat was inversely associated with eight different lipid classes including ceramides (Cer), gangliosides (GM3), diacylglycerols (DG), triacylglycerols (TG), lysoalkylphosphatidylcholine (LPC-O), alkylphosphatidylethanolamine (PE-O) and plasmalogens: alkenylphosphatidylcholine-P (PC-P) and alkylphosphatidylethanolamine-P (PE-P) (all $\mathrm{p}<0.05$ after $\mathrm{BH}$ correction; Figure 1).

- There were no associations between lipid classes or species and BMI, WC, or WHR (all p>0.05).

Fig 1. Correlations between body fat and lipid classes

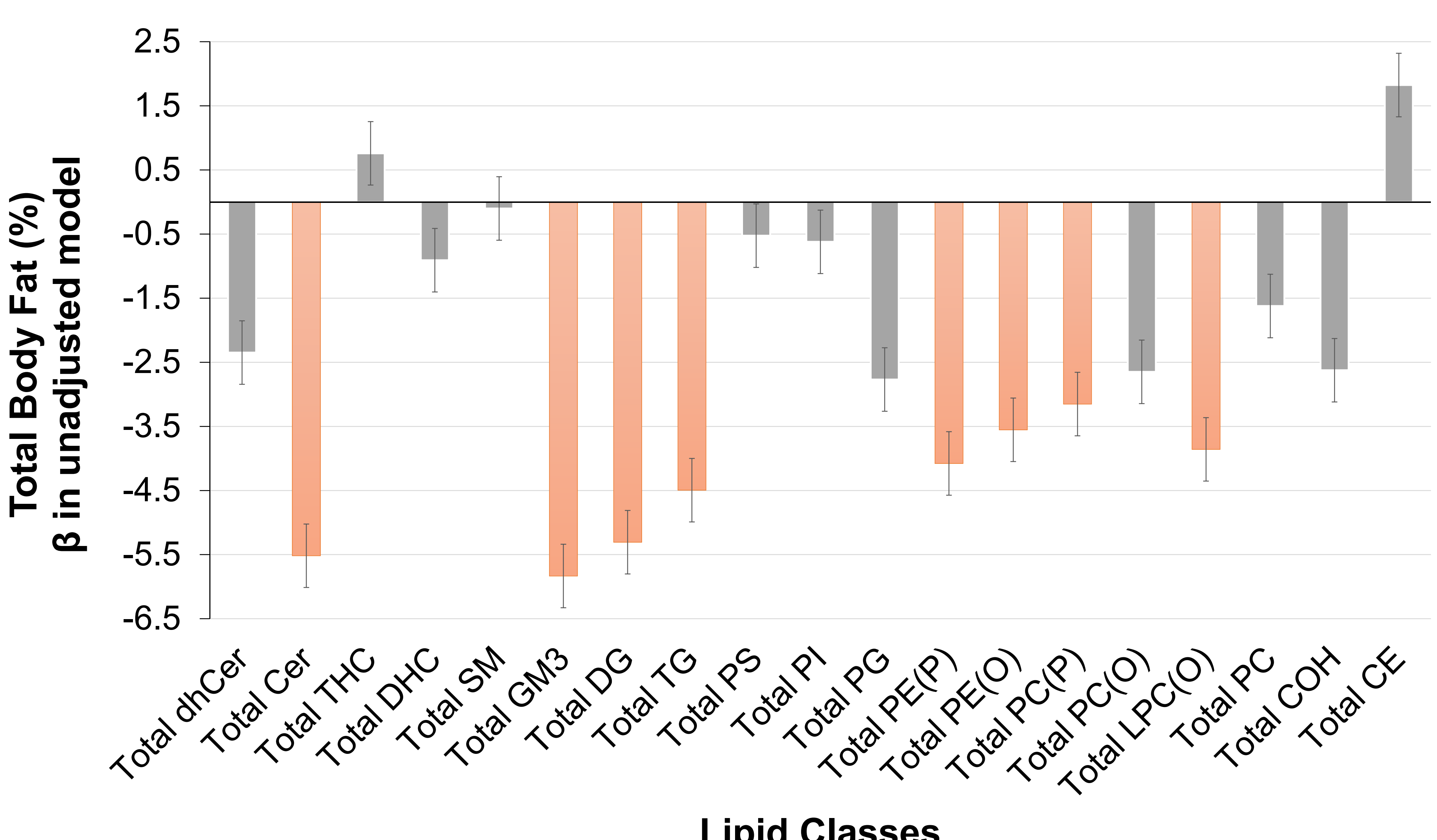

Fig 2. Relationship between body fat and lipids after adjustment for age and sex

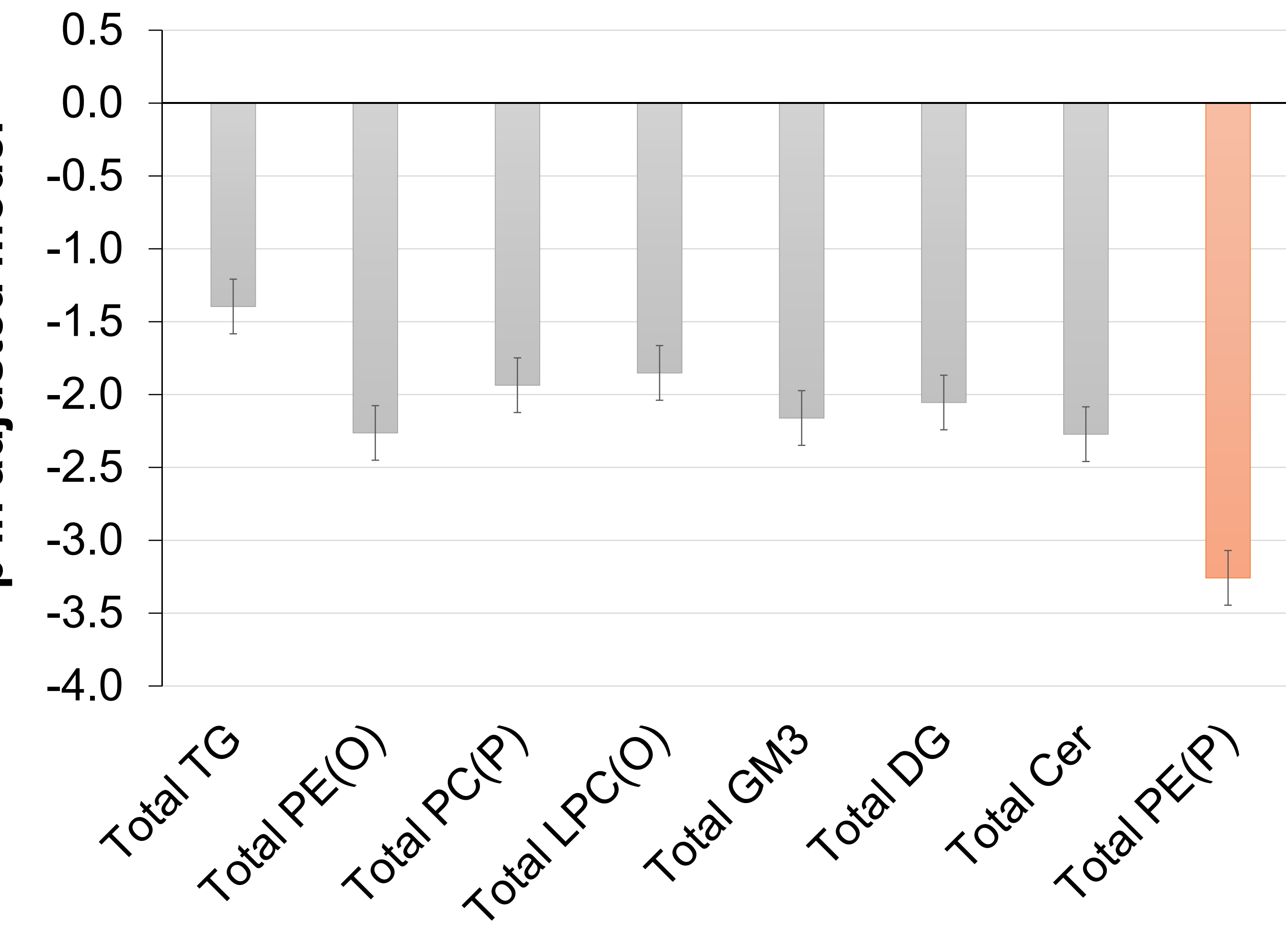

Lipid Classes

- After adjusting for age and sex, only the plasmalogen lipid class $\mathrm{PE}-\mathrm{P}$ remained inversely associated with total percent body fat $(\beta=-3.3, p=0.03$; Figure 2$)$.

\section{$\underline{\text { Conclusions }}$}

- Our findings are consistent with previous studies showing that plasmalogen lipid classes are reduced in obesity.

- However, most previous studies have used indirect anthropometric measures such as BMI or WHR.

- Our data suggest that greater adiposity determined by body fat is more strongly related to reduced plasmalogen concentrations than BMI, WC, or WHR in overweight or obese non-diabetic adults.

\section{References:}

- Weir, et al. J Lipid Res, 2013

- Tonks et al., Obesity, 2016

- Pietiläinen, et al., PlosOne, 2007

- Hanamatsu et al., Nutr.

Diabetes, 2014
Abbreviations:

$\mathrm{COH}$ : cholesterol

$\mathrm{CE}$ : cholesterol ester dhCer: dihydroceramides DHC: dihexosylceramides PC: phosphatidylcholine PC-O: alkylphosphatidylcholine PG: phosphatidylglycerol PI: phosphatidylinositol PS: phosphatidylserine SM: sphingomyelin THC: trihexosylcermides 